
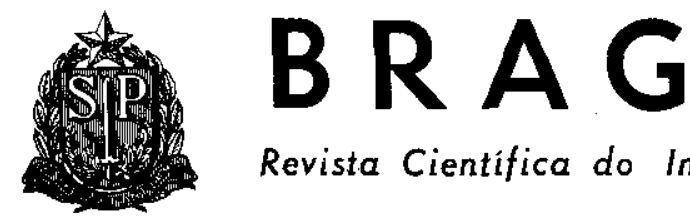

A

NT TA

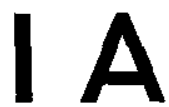

Revista Cientifica do Instituto Agronômico, Campinas

Vol. 41

Campinas, fevereiro de 1982

Artigo $n .^{0} 4$

\title{
DELINEAMENTOS COMPOSTOS CENTRAIS DUPLOS (1)
}

\author{
Armando Conagin, Divisão de Plantas Alimentícias Básicas, Instituto Agronômico
}

\section{RESUMO}

O presente artigo trata de um tipo de delineamento denominado "composto central duplo", basicamente simétrico, constituído de dois fatoriais (ou fatoriais fracionados) nos níveis $\pm 1 \mathrm{e} \pm \mathrm{W}$, duas estrelas nos niveis $\pm \alpha$ e $\pm \gamma \alpha$ para cada fator $\mathrm{e}$ pontos centrais, e podendo apresentar cinco, sete ou nove niveis para cada fator. Devido à maior flexibilidade desse tipo de delineamento em relação ao composto central, propriedades como ortogonalidade dos coeficientes, subdivisão ortogonal em blocos e rotacionalidade são mais facilmente integradas no mesmo delineamento. Como podem ser estabelecidos com uma parte fracionada de um fatorial completo, esses delineamentos podem explorar um maior número de níveis na amplitude de variação que se pretende pesquisar, com um número um pouco maior de pontos que o delineamento composto central correspondente. Na Agricultura, em contraste com os experimentos tecnol6gicos e, principalmente, nas pesquisas com fertilizantes, devido a variabilidade do solo e do clima, os experimentos devem ser efetuados com a utilizaçăo dos tratamentos do delineamento colocados, todos, de uma so vez. As respostas obtidas podem ser mais altas, na amplitude estudada, nos anos bons, e, nos anos ruins, de menor magnitude, ascendente e com resposta em uma amplitude menor, apresentando, em certos casos, uma "resposta em plató" para as dosagens mais altas. $O$ delineamento composto central duplo pode adaptar-se melhor a essa peculiaridade, porque, pela sua estrutura, utiliza maior número de pontos na amplitude de dosagens utilizada. Se uma calibração adequada foi originalmente adotada e o ano agrícola muito bom, a amplitude maior utllizada capta a resposta aos fertilizantes em toda a extensão pesquisada; se o ano foi ruim, a resposta aos fertilizantes é de menor magnitude e, provavelmente, com resposta da "curva em plató" para as dosagens mais altas; a análise do delineamento como um todo pode causar, nesse caso, um "viés" nos coeficientes do modelo, e a determinaçäo da dosagem econômica fica prejudicada; nesse caso, o delineamento proposto torna possível contornar o problema, efetuando a análise da parte central do delineamento como um delineamento composto central simples, usando os níveis \pm 1 para a parte fatorial e $\pm \alpha$ para a axial, possibilitando a obtenção de uma estimativa justa (não enviesada) dos coeficientes do modelo e uma determinação mais precisa das dosagens econômicas; para a mesma amplitude estudada, isso não poderia ser obtido caso se utilizasse um delineamento compasto central ou um fatorial $3 \times 3 \times 3$. Basica-

(1) Trabalho apresentado na 10.a Conferência Internacional de Biometria, Guarujá (SP), em agosto de 1979. Recebido para publicação a 24 de junho de 1980. 
mente, este artigo apresenta três tipos de delineamento; no quadro 1, delineamentos que, além de ortogonais, são subdivisíveis em dois, três ou cinco blocos: os niveis foram escolhidos de forma a que os níveis $W$ da parte fatorial sejam menores que dois (níveis mais altos são raramente utilizados na prática). Os delineamentos do segundo grupo (Quadro 2) sáo ortogonais, divisíveis ortogonalmente em blocos, com os pontos da parte fatorial inseridos em duas hiperesferas de raios $\alpha=\sqrt{\mathbf{k}} \mathrm{e} \gamma \alpha \approx \mathrm{W} \sqrt{\mathrm{k}}$, contendo dois ou quatro pontos centrais; esses delineamentos podem ser considerados como constituídos de dois delineamentos compostos centrais, apresentando, cada um deles, segundo LUCAS $(\boldsymbol{y})$, eficiência do tipo D-ótimo. No terceiro grupo, incluídos no quadro 3 , os delineamentos são ortogonais, subdivisíveis ortogonalmente em blocos e com rotacionalidade plena ou quase plena (marcados com um asterisco), no sentido dado por BOX \& HUNTER (1), apresentado com um número adequado de pontos centrais, visando fornecer boa estimativa do erro experimental e possibilitando, assim, um teste mais preciso de adequação do modelo estudado. Esses delineamentos podem ser de utilidade aos pesquisadores e aos estatísticos naqueles casos, como na pesquisa agrícola e na pequisa com fertilizantes, em que a subdivisăo em blocos é muito importante; possibilitam uma determinação precisa da dose econômica dos fertilizantes e servem também para aqueles casos em que se sente que é importante cobrir a área pesquisada com várias doses de cada fator.

\section{INTRODUÇÃO}

Os delineamentos compostos centrais têm sido utilizados de forma crescente na pesquisa moderna, não só no campo industrial como em outras áreas.

Constam, basicamente, de uma parte fatorial (ou fração do fatorial), de pontos axiais nos níveis $-\alpha \mathrm{e}+\alpha$ de cada fator e de pontos localizados no centro do delineamento. Assim, para três fatores $(k=3)$, necessitam de um número mínimo de quinze pontos, sendo oito pertencentes a um fatorial $2 \times 2 \times 2$; - a parte axial consta de seis pontos (dois pontos em cada eixo), além de um ponto central.

Esses delineamentos podem ser ortogonais ou não, rotacionais ou não, ou simultaneamente ortogonais e rotacionais, podendo todos eles ser ou não subdivisíveis em blocos. A teoria sobre esses delineamentos e o desenvolvimento dos vários tipos é devida a BOX \& WILSON (2), BOX \& HUNTER (1) e outros.

Normalmente prestam-se bem para estudos de superfície de res- posta; o modelo utilizado pode ser mais simples (de primeiro grau) ou mais complexo (de segundo, terceiro grau etc.).

VOSS \& PESEK (10), visando efetuar estudos de adubação com a análise econômica dos resultados, apresentaram em 1967 um delineamento para o estudo de três fatores $(\mathrm{k}=3$ ), composto de dois cubos com oito pontos cada um, nos níveis $\pm 1 \mathrm{e}$ \pm 2 , seis pontos axiais nos níveis \pm 2 e um ponto central, num total de 23 pontos.

Os delineamentos "compostos centrais duplos" constam de dois conjuntos fatoriais (ou fatoriais fracionados) nos níveis $\pm 1 \mathrm{e} \pm \mathrm{W}$, de um número duplo de pontos axiais (nos níveis $\pm \alpha$ e $\pm \gamma \alpha$ para cada fator) e de certo número de pontos centrais.

Os compostos centrais duplos possibilitam, para $\mathrm{k}$ fatores, adaptar-se uma superfície de resposta através de vários níveis de cada fator (de cinco a nove), com utilização de um número razoavelmente reduzido de pontos. 
Esses delineamentos apresentam maior flexibilidade do que os compostos centrais, pelas seguintes razões: podem explorar maior gama de dosagens dentro da amplitude considerada; permitem mais facilmente a obtenção dos tipos ortogonais, rotacionais ou ambos, e ainda a partição ortogonal em blocos de forma mais fácil, qualidades bastante desejáveis para os delineamentos em geral (1).

\section{MATERIAL E METODO}

De forma semelhante ao utilizado no composto central, o modelo geral para estudo do composto central duplo, com a inclusão de blocos, é o que se segue (9):

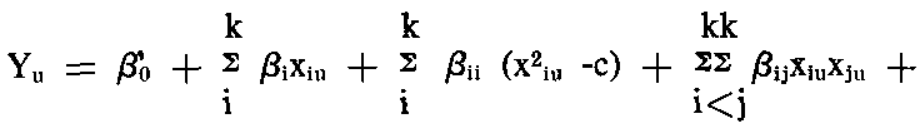

$$
\begin{aligned}
& \text { b } \\
& +\Sigma \delta_{m}\left(z_{m u}-\bar{z}_{m}\right)+\epsilon_{\mathfrak{u}} \\
& \text { m }
\end{aligned}
$$

onde:

$$
\begin{aligned}
& \mathrm{u}=1,2, \ldots, \mathrm{N} \\
& \mathrm{i}, \mathrm{j}=1,2, \ldots, \mathrm{k} \\
& \mathrm{m}=1,2, \ldots, \mathrm{b} .
\end{aligned}
$$

Como exemplo, a matriz $\mathrm{X}$ das observações, para $\mathbf{k}=2, \mathrm{~N}=18$,

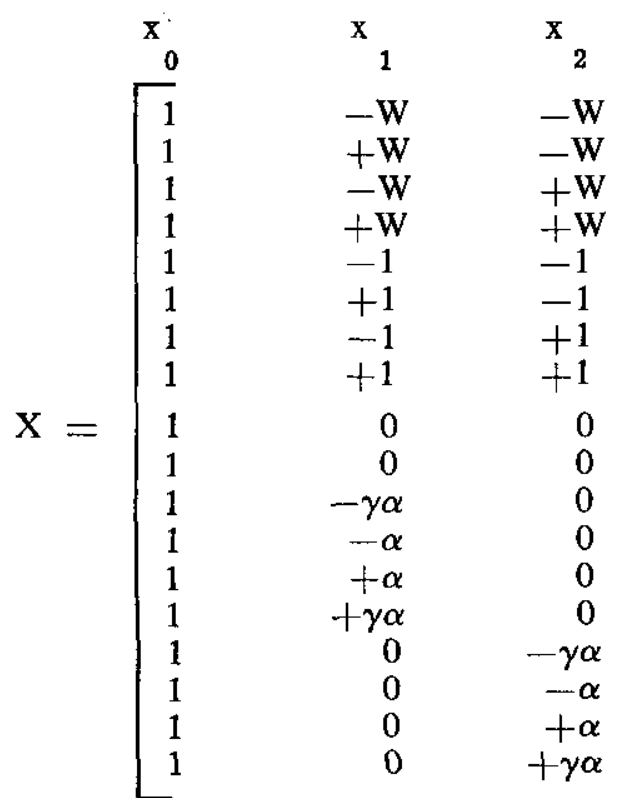

$\mathrm{n}=2$ (onde $\mathrm{N}$ é o número total de observaçốes e $\mathrm{n} \alpha$ número de pontos centrais) é a dada a seguir; a parte referente a blocos, nos casos da subdivisão ortogonal em blocos, é calculada isoladamente.

$\left.\begin{array}{rrr}\mathrm{X}^{2}{ }-\mathrm{c} & \mathrm{X}^{2}{ }^{2}-\mathrm{c} & \mathrm{x}_{1} \mathrm{X}_{2} \\ & & \\ \mathrm{~W}^{2}-\mathrm{c} & \mathrm{W}^{2}-\mathrm{c} & \mathrm{W}^{2} \\ \mathrm{~W}^{2}-\mathrm{c} & \mathrm{W}^{2}-\mathrm{c} & -\mathrm{W}^{2} \\ \mathrm{~W}^{2}-\mathrm{c} & \mathrm{W}^{2}-\mathrm{c} & -\mathrm{W}^{2} \\ \mathrm{~W}^{2}-\mathrm{c} & \mathrm{W}^{2}-\mathrm{c} & \mathrm{W}^{2} \\ 1-\mathrm{c} & 1-\mathrm{c} & 1 \\ 1-\mathrm{c} & 1-\mathrm{c} & -1 \\ 1-\mathrm{c} & 1-\mathrm{c} & -1 \\ 1-\mathrm{c} & 1-\mathrm{c} & 1 \\ -\mathrm{c} & -\mathrm{c} & 0 \\ -\mathrm{c} & -\mathrm{c} & 0 \\ \gamma^{2} \alpha^{2}-\mathrm{c} & -\mathrm{c} & 0 \\ \alpha^{2}-\mathrm{c} & -\mathrm{c} & 0 \\ \alpha^{2}-\mathrm{c} & -\mathrm{c} & 0 \\ \gamma^{2} \alpha^{2}-\mathrm{c} & -\mathrm{c} & 0 \\ -\mathrm{c} & \gamma^{2} \alpha^{2}-\mathrm{c} & 0 \\ -\mathrm{c} & \alpha^{2}-\mathrm{c} & 0 \\ -\mathrm{c} & \alpha^{2}-\mathrm{c} & 0 \\ -\mathrm{c} & \gamma^{2} \alpha^{2}-\mathrm{c} & 0 \\ & & \end{array}\right]$


Nessa matriz, 1 e W representam os níveis da parte fatorial, $\alpha$ e $y \alpha$ os da parte axial e c, o valor médio dos coeficientes correspondentes aos termos quadráticos puros $\left(\beta_{i i}\right)$. A extensão de $\mathrm{k}=2$ para $\mathrm{k}=3$, 4 etc. é facilmente perceptível.

$$
\begin{aligned}
& \text { A matriz } \mathbf{A}=\mathbf{X}^{\prime} \mathrm{X} \text { para } 0 \text { caso } \mathbf{k}=2 \text { (mais simples) é: } \\
& \mathbf{A}=\mathrm{X}^{\prime} \mathrm{X}=\left[\begin{array}{cccccc}
\mathrm{N} & 0 & 0 & 0 & 0 & 0 \\
0 & \mathrm{~d} & 0 & 0 & 0 & 0 \\
0 & 0 & \mathrm{~d} & 0 & 0 & 0 \\
0 & 0 & 0 & \mathrm{p} & \mathrm{q} & 0 \\
0 & 0 & 0 & \mathrm{q} & \mathrm{p} & 0 \\
0 & 0 & 0 & 0 & 0 & \mathrm{~h}
\end{array}\right]
\end{aligned}
$$

A matriz $\mathrm{A}$ para $\mathrm{k}=3$, 4 etc. é facilmente determinável.

A teoria aqui apresentada para os delineamentos simétricos, do tipo composto central duplo, representa uma extensão daquela desenvolvida por BOX \& WILSON (2) e por BOX \& HUNTER (1) para os delineamentos compostos centrais.

Para $\mathrm{k}$ fatores, tem-se:

$$
\begin{aligned}
& \mathrm{N}=2^{\mathrm{k}-\mathrm{f}}+2^{\mathrm{k}-\mathrm{f}}+2 \mathrm{k}+2 \mathrm{k}+\mathrm{n} \\
& \mathrm{d}=\mathrm{F}^{\prime}\left(1+\mathrm{W}^{2}\right)+2 \alpha^{2}\left(1+\gamma^{2}\right), \text { onde } \mathrm{F}^{\prime}=2^{\mathrm{k}-\mathrm{f}} \\
& \mathrm{p}=\mathrm{F}^{\prime}\left(\mathrm{W}^{2}-\mathrm{c}\right)^{2}+\mathrm{F}^{\prime}(1-\mathrm{c})^{2}+2\left(\alpha^{2}-\mathrm{c}\right)^{2}+2\left(\gamma^{2} \alpha^{2}-\mathrm{c}\right)^{2}+(\mathrm{T}-4) \mathrm{c}^{2} \\
& \mathrm{q}=\mathrm{F}^{\prime}\left(\mathrm{W}^{2}-\mathrm{c}\right)^{2}+\mathrm{F}^{\prime}(1-\mathrm{c})^{2}-4 \mathrm{c}\left(\alpha^{2}-\mathrm{c}\right)-4 \mathrm{c}\left(\gamma^{2} \alpha^{2}-\mathrm{c}\right)+(\mathrm{T}-8) \mathrm{c}^{2} \\
& \mathrm{~h}=\mathrm{F}^{\prime}\left(1+\mathrm{W}^{4}\right) \\
& \mathrm{c}=\left[\mathrm{F}^{\prime}\left(1+\mathrm{W}^{2}\right)+2 \alpha^{2}\left(1+\gamma^{2}\right)\right] \div \mathrm{N}=\mathrm{d} \div \mathrm{N} \\
& \mathrm{T}=2 \mathrm{k}+2 \mathrm{k}+\mathrm{n}, \quad \mathrm{N}=2 \mathrm{~F}^{\prime}+\mathrm{T} .
\end{aligned}
$$

$\mathrm{Na}$ fórmula $\mathrm{F}^{\prime}=2^{\mathrm{k}-\mathrm{f}}, \mathrm{f}$ indica o grau de fracionamento; por exemplo, $\operatorname{com} \mathrm{f}=0$, o fatorial é completo, com $\mathrm{f}=1$ tem-se metade do fatorial, com $\mathrm{f}=2$, a quarta parte etc.

É facilmente demonstrável que $\mathrm{p}=\mathrm{q}+2 \alpha^{4}\left(1+\gamma^{4}\right)$.

Um delineamento desse tipo, para $\mathrm{k}=3$, não ortogonal, com $\alpha= \pm 1, \gamma \alpha= \pm 2$, fatoriais nos níveis 1 e 2 , um ponto central, totalizando $\mathrm{N}=29$ pontos (cinco níveis para cada fator) foi desenvolvido para estudos de adubação NPK por CONA-
GIN et alii (4) em cujo trabalho se encontram $o$ cálculo das estimativas dos parâmetros $\beta$, a análise da variância e o valor dos coeficientes do modelo e suas variâncias.

\subsection{Compostos Centrais Duplos, Ortogonais}

Se se impuser a condição de que q seja tornado nulo, as covariâncias entre os parâmetros $\beta_{\text {ii }}$ tornam-se nulas e a matriz $A=X^{\prime} X$ se transforma em uma matriz diagonal; nesse caso os parâmetros $\beta$ são estimados independentemente (3). 


\subsection{Compostos Centrais Duplos, Ortogonais e Divisiveis em Blocos}

Para se obter a partição ortogonal em blocos, deve-se aplicar, para esses delineamentos, as mesmas condições estipuladas por BOX \& HUNTER (1) e BOX \& WILSON (2), para os compostos centrais; para isso, resumidamente, é necessário que cada bloco se constitua em um delineamento de primeira ordem, ortogonal, e, ainda, que a contribuição de cada bloco para o total da soma de quadrados do delineamento seja proporcional ao número de unidades existentes dentro de cada bloco (2); uma exposição detalhada de como proceder, nesses casos, é dada por MYERS (9).

A solução generalizada para ortogonalização e partição em blocos ortogonais do composto central duplo, aplicando as condições:

$$
\begin{aligned}
& \text { a) } q=0 \\
& \text { b) } \frac{\Sigma x^{2}{ }_{i n}\left(\text { axial }+a_{0}\right)}{\Sigma x^{2}{ }_{t u}(\text { total })}=\frac{n_{n}}{N} \text {, }
\end{aligned}
$$

é a seguinte:

$$
\begin{aligned}
& 2 Z^{2} \alpha^{4}\left\{\left(N-n_{a}\right)^{2}-N F\right\}-2 Z \alpha^{2}\left\{n_{a} F^{\prime}\left(N-n_{n}\right)\right\}+n_{a}^{2} F^{\prime 2}=0 \\
& \text { onde } Z=\left(1+\gamma^{2}\right) .
\end{aligned}
$$

Nessas condições, $a_{0}$ representa o número de pontos centrais incluídos na parte axial; o número total de pontos centrais no delineamento é, portanto, $\pi=a_{0}$ (axial) $+b_{0}$ (fatorial). Ainda, $\mathrm{n}_{\mathrm{g}}$ representa a soma dos pontos do bloco axial, estando aí incluídos os $\mathrm{a}_{0}$ pontos centrais.

Como $\mathrm{N}, \mathrm{F}, \mathrm{n}_{\mathrm{a}}$ e $\alpha$ são valores escolhidos, resolvendo-se a equação (II) chega-se a Z, $\gamma$ e, a seguir, a W (nível do segundo fatorial), onde:

$\mathrm{W}^{2}=\left[\frac{2\left(\mathrm{~N}-\mathrm{n}_{\mathrm{H}}\right)}{\mathrm{n}_{\mathrm{a}} \mathrm{F}^{\prime}} x^{2}\left(1+\gamma^{2}\right)\right]-1$

Determinados os valores $\gamma, \gamma \alpha$ e $\mathrm{W}$, pode-se verificar que $\mathrm{q}=0 \mathrm{e}$ que a condição de ortogonalidade de blocos, dada por:

$$
\frac{2 \alpha^{2}\left(1+\gamma^{2}\right)}{\mathrm{F}^{\prime}\left(1+\mathrm{W}^{2}\right)+2 \alpha^{2}\left(1+\gamma^{2}\right)}=\frac{\mathrm{n}_{\mathrm{a}}}{\mathrm{N}},
$$

também se verifica.
Os níveis para cada um dos fatores em estudo são: ${ }^{ \pm} 1 \mathrm{e} \pm \mathrm{W}$ para a parte fatorial; $\pm_{\alpha}$ e ${ }^{ \pm} \gamma \alpha$ para a parte axial e zero para o valor central, isto é, existem nove níveis para cada um dos $\mathbf{k}$ fatores. Dependendo de outras condições impostas, como $\alpha=1$, por exemplo, os nove níveis podem ser reduzidos para sete; restrição adicional pode reduzi-lo para cinco, ficando difícil, então, a obtenção da ortogonalidade e a partição em blocos.

\subsubsection{Resultados do quadro 1}

Os resultados para $\mathrm{k}$ fatores, fazendo-se variar $\mathbf{k}$ de dois a sete, com repetição completa dos fatoriais ( $=0$ ), metade dos tratamentos ( $f=1$ ) ou a quarta parte dos mesmos ( $f=2$ ). encontram-se no qua dro 1.

Os valores de $\alpha$ escolhidos foram tais que proporcionaram valores de 
W entre 1,5 e 2,0 , valores considerados altamente convenientes para a exploração da parte fatorial da superfície de resposta.

Um caso especial desse tipo de delineamento com as características $\mathrm{k}=3, \mathrm{~W}=4,391, \alpha=3,631$, $\gamma=2$, partição em dois blocos, com o ponto central localizado no bloco dos axiais foi apresentado por CONAGIN \& JORGE, acompanhado de um exemplo, com todos os cálculos (3).

2.2.2 Resultados do quadro 2

Os resultados dessa tabela correspondem a $\mathrm{k}$ variando de dois a sete e foram calculados impondo-se as seguintes condições: 1) que $\alpha=\sqrt{\mathrm{k}}$ e que $\gamma \approx \mathrm{W} ; 2$ ) que o número de pontos centrais fosse dois ou quatro, possibilitando a subdivisão em blocos; neste caso buscou-se obter um delineamento que fosse composto de dois compostos centrais do tipo quase D-ótimo com os raios de hiperesfera nos níveis $\sqrt{\mathrm{k}}$ e $W \sqrt{\mathrm{k}}(7)$ e (8).

\subsection{Compostos Centrais Duplos, Rotacionais}

As condições necessárias para a obtenção deste tipo são semelhantes àquelas desenvolvidas por $\mathrm{BOX} \&$ HUNTER (1) para os compostos centrais rotacionais. Para se obter rotacionalidade, isto é, a uniformidade de variâncias para os pontos eqüidistantes do centro, no caso dos delineamentos em que se utilizam modelos do segundo grau, há necessidade, de acordo com BOX \& HUNTER (1) e MYERS (9), de que

$$
\Sigma_{\mathrm{X}_{\mathrm{iu}}{ }^{4}}=3 \Sigma_{\mathrm{iu}}^{\mathrm{X}_{\mathrm{ju}}^{2}} \mathrm{X}^{2}
$$

Essa condição, aliada à condição de ortogonalidade $(\mathrm{q}=0)$, levar-nos-ia à obtenção de delineamentos compostos centrais duplos, ortogonais e rotacionais.

\subsection{Compostos Centrais Duplos, Ortogonais, Divisíveis em Blo- cos e Rotacionais}

Para a obtenção desse último tipo, deve-se fazer simultaneamente com que
a) $\mathrm{q}=0$;
b)

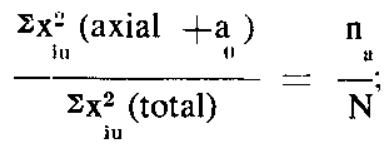

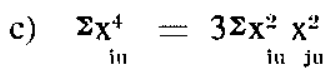

No caso do composto central duplo, a condição c torna-se:

$$
\begin{aligned}
\mathrm{F}^{\prime} & \left(1+\mathrm{W}^{4}\right)+2 \alpha^{4}\left(1+\gamma^{4}\right)= \\
& =3 \mathrm{~F}^{\prime}\left(1+\mathrm{W}^{4}\right) \text { ou } \\
\alpha & =\left[\frac{\mathrm{F}^{\prime}\left(1+\mathrm{W}^{4}\right)}{\left(1+\gamma^{4}\right)}\right]^{1 / 4}
\end{aligned}
$$

2.4.1 Resultados do quadro 3

Utilizando-se as condições de ortogonalidade, partição ortogonal em blocos e rotacionalidade, obtiveram-se os resultados constantes do quadro 3. A obtenção de rotacionalidade implica na necessidade de alocação de um número maior de pontos centrais que os necessários para a obtenção das soluções constantes dos quadros 1 e 2 . Mas, com isso, pode-se obter melhor estimativa do erro e assim testar de forma mais precisa a adequação do modelo adotado.

No quadro 3 os delineamentos ou são perfeitamente rotacionais ou quase rotacionais (assinalados com um asterisco), calculados com $F^{\prime}$ completo ( $\mathrm{f}=0$ ), metade ( $\mathrm{f}=1$ ) ou com a quarta parte do fatorial $(f=2)$. 


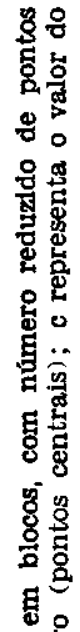

8 음

\section{|}

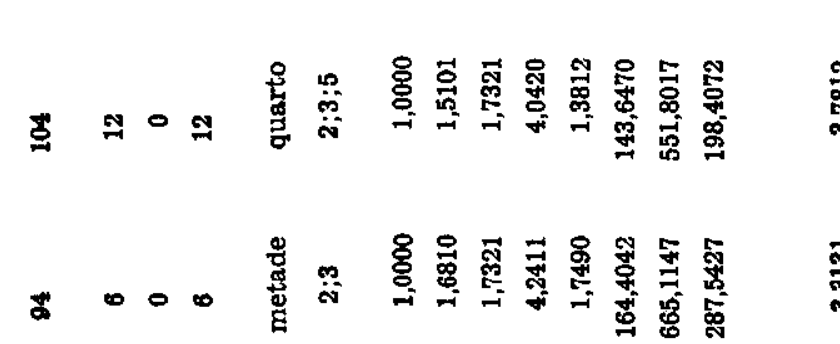
$\underset{\infty}{\infty}$ $\$$ $\mathbf{4}$
4 案 용 용 퉝 果 $\sum_{0}$ 공 $\downarrow$ क 웜 है 용 苟 농

ำ

을 용

하요요

焉羿

영요

o

$\infty$

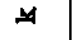

\& $\infty \circ \infty$

竞

क

is

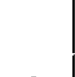

10
11

○ $\infty \circ \infty$

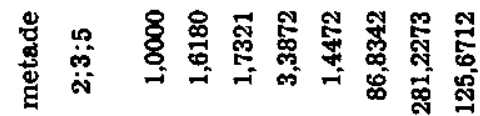

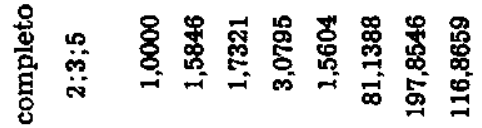

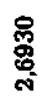

冎 $\infty 00$

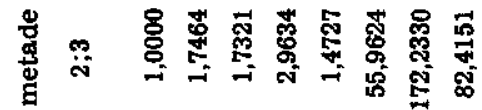

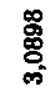

กิ $+0+$

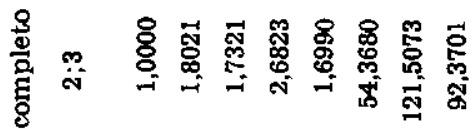

花

误需:

就

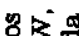

足朴

运获要

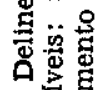

, 空

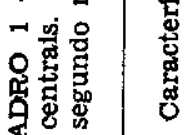

总

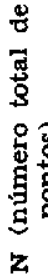

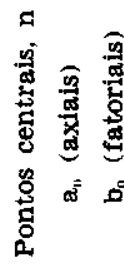

壱

음

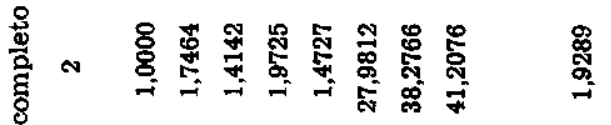

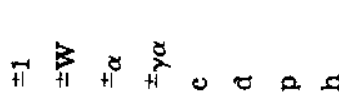

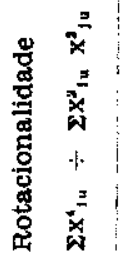




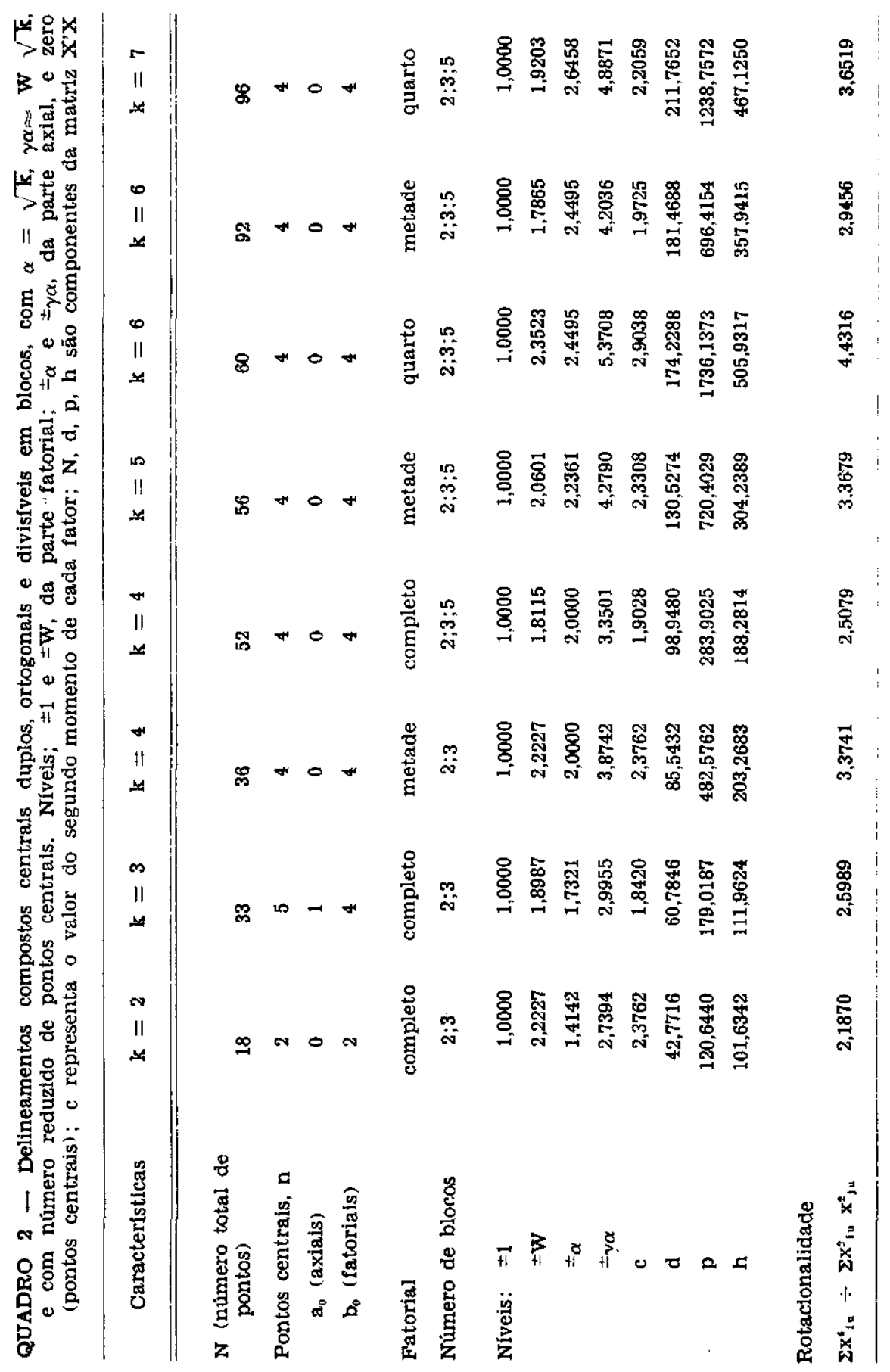




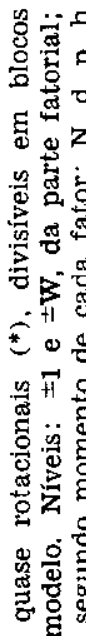

눙유

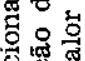

誌

옹요

F

엻

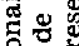

路绻

荡

\%

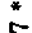

ஜ

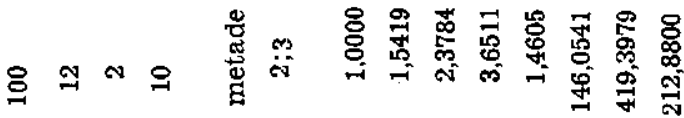

홍

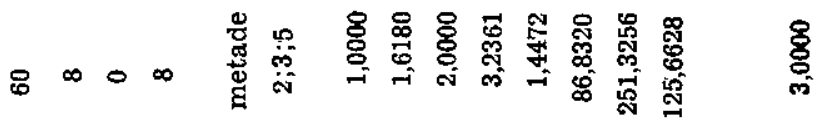

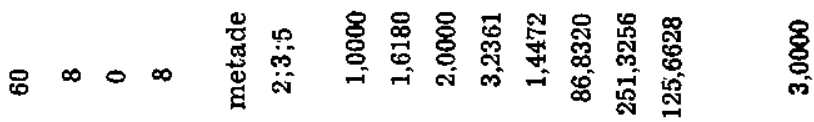

10
11

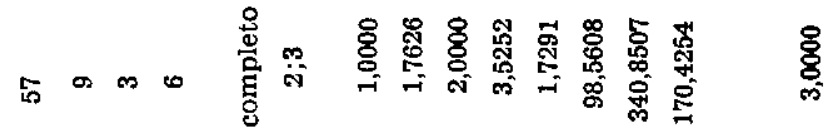

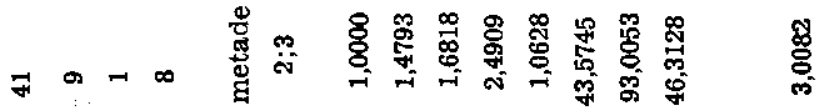

总密密

.

善焉

융

5

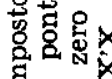

宕守

象要要

要

究哭

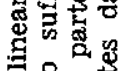

岁氙卷

11

1 总。

$\infty$ +ो

용 8

营

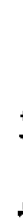

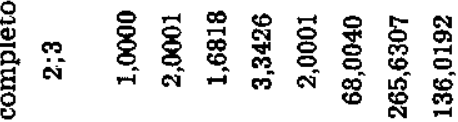

突

मेळの

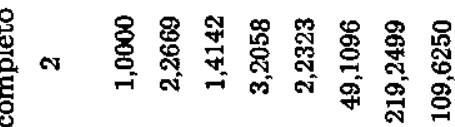

छ

官

莺

㶾

要

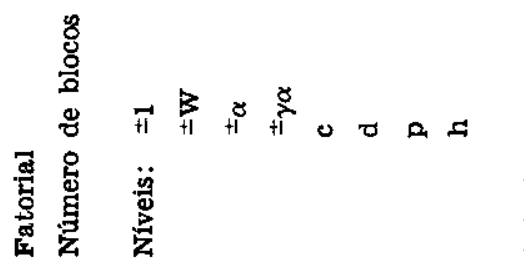

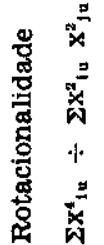




\subsection{Alocação dos Tratamentos nos Blocos}

A alocação dos tratamentos da parte axial é feita sempre colocando-os em um dos blocos. A parte fatorial pode ser dividida em dois ou quatro blocos ou até mais, dependendo do fracionamento da parte fatorial e do(s) confundimento(s) adotado(s). No caso de $\mathbf{k}=3$, do Quadro 1 , em que o fatorial está completo, é desejável efetuar o confundimento da interação tripla e dividir a parte fatorial em dois blocos.

Quando os delineamentos são ortogonais e divisíveis ortogonalmente em blocos, a soma de quadrados destes é calculada facilmente, à parte e independentemente da matriz $\mathrm{X}^{\prime} \mathrm{X}$.

No caso de partição em três blocos, um bloco é o da parte axial $B_{a} e$ dois blocos são os da parte fatorial $\mathbf{B}_{1}$ e $\mathbf{B}_{2}$; seu calculo é feito assim:

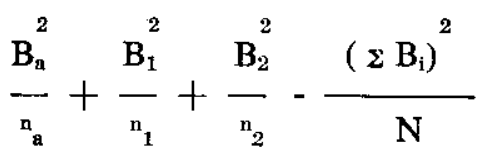

onde ${ }_{a}=2 k+2 k+a_{0}$

$\mathrm{e} \mathrm{n}_{1}=\mathrm{n}_{2}=\frac{\mathrm{F}^{\prime}}{2}+\frac{\mathrm{F}^{\prime}}{2}+\frac{\mathrm{b}_{0}}{2}$.
No caso de cinco blocos, tere$\operatorname{mos} \mathrm{n}_{1}=\mathrm{n}_{2}=\mathrm{n}_{3}=\mathrm{n}_{4}$ sendo $\mathrm{n}_{1}=\frac{\mathrm{F}^{\prime}}{4}+\frac{\mathrm{F}^{\prime}}{4}+\frac{\mathrm{b}_{0}}{4} \mathrm{e}$ $\mathrm{n}_{\mathrm{a}}$ definido como anteriormente.

Para se obter o melhor confundimento para a parte fatorial (ou fatorial fracionado) visando à partição em blocos, devem-se escolher as interações de nível elevado que sejam de pouca importância e cujos "aliases" não envolvam interações de dois elementos (se possível) ou os efeitos principais. Já existem soluções bem estabelecidas em DAVIES (5) e em HARTLEY (6).

\subsection{Análise da Variância}

A análise da variância é feita de forma muito semelhante à do composto central; exemplos deste último tipo encontram-se em DAVIES (5) e em MYERS (9).

A solução $\hat{\beta}=A^{-1} X^{\prime} \underline{Y}$ leva à obtenção das estimativas dos coeficientes do modelo. Como, porém, o delineamento é ortogonal, cada coeficiente é estimado direta e independentemente.

A soma de quadrados devida à regressão polinomial é obtida de forma muito simples:

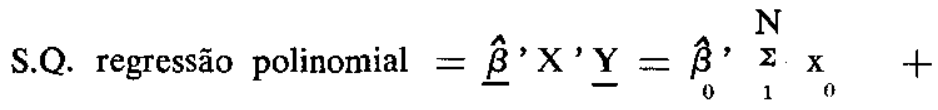

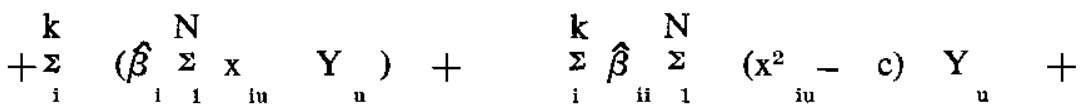

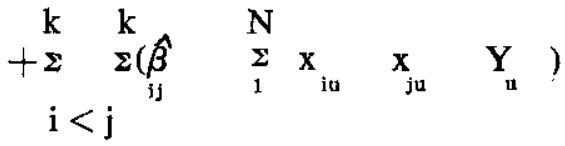


Cada componente da soma de quadrados, dividido por um grau de liberdade, proporciona o quadrado médio correspondente ao coeficiente em estudo. Os componentes podem, então, ser testados, individualmente, em relação ao quadrado médio do resíduo. Este contém $N-p-(b-1)$ graus de liberdade, onde $\mathrm{N}$ representa o número de observações experimentais incluídas no delineamento, $\mathrm{p}$ o número de parâmetros da equação polinomial adotada $\mathrm{e} b$ o número de blocos do delineamento. Maiores detalhes sobre a análise da variância, com a análise completa de um exemplo para o composto central duplo, encontra-se em CONAGIN \& JORGE (3).

Efetuado o teste em função de um nível $\alpha \%$ (erro tipo I), preestabelecido, pode-se calcular o valor esperado $\hat{Y}_{\mathrm{u}}$, reescrevendo o modelo como em (I), com a diferença de manter, no modelo, só os termos significativos, também excluindo do mesmo o componente de blocos, de forma a que se obtenham valores $\hat{\mathbf{Y}}_{\mathrm{u}}$, já ajustados para blocos.

\subsection{Utilização dos quadros}

Os dados constantes dos quadros possibilitam aos usuários a utilização direta dos delineamentos propostos, pois suas características mais importantes encontram-se incluídas nos mesmos.

No caso de se estudarem três fatores $(\mathrm{k}=3)$ e se tiver escolhido o delineamento que tinha as propriedades inerentes às contir.as no quadro 3, ter-se-á: $k=3, N=34$, fatorial completo, com partição em três blo$\cos , \mathrm{n}=6$, sendo $\mathrm{a}_{\mathrm{o}}=2$ e $\mathrm{b}_{\mathrm{o}}=4$; nesse caso a interação $\mathrm{ABC}$ deverá ser, de preferência, a interação confundida para a partição da parte fatorial em dois blocos (com dois pontos centrais em cada um).

O bloco axial terá $6+6+2=$ $=14$ pontos e os outros dois blocos,

$$
\underset{\mathrm{um} .}{\frac{8}{2}}+\frac{8}{2}+2 \text {, isto é, } 10 \text { pontos cada }
$$

Os níveis dos fatores são os seguintes: $\pm 1,0000 \mathrm{e} \pm 2,0001$ para a parte fatorial; os valores axiais serão $\alpha=1,6818$ e $\gamma \alpha=3,3426$, sendo $\mathrm{c}=2,0001$.

Usando-se as fórmulas básicas apresentadas e lembrando que $\mathrm{N}=34, \mathrm{~F}^{\prime}=8, \mathrm{k}=3, \mathrm{a}_{0}=2$, $\mathrm{b}_{0}=4, \mathrm{n}_{\mathrm{a}}=14$, têm-se:

$$
\begin{aligned}
& \mathrm{d}=\mathrm{F}^{\prime}\left(1+\mathrm{W}^{2}\right)+2 \alpha^{2}\left(1+\gamma^{2}\right)= \\
& =68,0040 \\
& \mathrm{p}=2 \alpha^{4}\left(1+\gamma^{4}\right)=265,6307 \\
& \mathrm{~h}=\mathrm{F}^{\prime}\left(1+\mathrm{W}^{4}\right)=136,0192 \\
& \mathrm{c}=\mathrm{d} \div \mathrm{N}=2,0001 \\
& \Sigma_{\mathrm{Xiu}}^{4} \div \underset{\Sigma \mathrm{XiuXju}}{2}=(\mathrm{p}+\mathrm{h}) \div \mathrm{h}= \\
& =2,9529
\end{aligned}
$$

As estimativas das variâncias dos coeficientes estimados são:

$$
\begin{aligned}
& \hat{v}_{\hat{\beta}_{\mathrm{i}}}=\mathrm{s}^{2} \div 68,0040 \\
& \hat{v}_{\hat{\beta}_{\mathrm{if}}}=\mathrm{s}^{2} \div 265,6307 \\
& \hat{\mathrm{v}}_{\hat{\beta}_{\mathrm{ij}}}=\mathrm{s}^{2} \div 136,0192 \text { etc. }
\end{aligned}
$$

onde $s^{2}$ é a estimativa do resíduo. 


\section{VANTAGENS DOS DELINEAMENTOS COMPOSTOS CENTRAIS DUPLOS}

A pesquisa tecnológica, em face da repetibilidade dos resultados $e$ do erro experimental reduzido, pode normalmente ser executada de forma sequiencial, possibilitando um direcionamento dos tratamentos sucessivos no sentido da otimização do processo.

Já a pesquisa agronômica de campo, devido às variações existentes de clima e solo, requer que a experimentação seja efetuada, toda ela, de uma só vez. Nos casos de experimentos de adubação, as respostas podem variar de tipo de solo para tipo de solo e de um ano para outro; normalmente, as respostas são mais acentuadas e crescem para uma amplitude maior de dosagens nos anos favoráveis; nos desfavoráveis, obtêm-se respostas menores e para uma amplitude menor de dosagens, havendo uma resposta em platô para as dosagens mais altas.

Usando esse tipo de delineamento e efetuando uma boa calibração das dosagens (escolha adequada da amplitude estudada), podem-se detectar as respostas até os níveis mais altos nos anos favoráveis e a resposta menor, "em platô", nos anos ruins. Neste último caso, ele pode fornecer estimativas dos coeficientes com certo "viés". Como é composto de dois centrais compostos, o delineamento pode ser plenamente utilizado no ano favorável e se pode, se for julgado necessário, eliminar o inconveniente citado fazendo a análise só do composto central nos níveis 1 e $\alpha$, nos anos desfavoráveis, eliminando o viés e obtendo melhor estimativa da do- sagem econômica. Nesse sentido, o composto central duplo apresenta uma vantagem adicional muito importante, pois nem o composto central nem os fatoriais $(3 \times 3 \times 3$, por exemplo), para a mesma amplitude, teriam a mesma flexibilidade.

\section{CONCLUSÕES}

Os delineamentos compostos centrais duplos são mais flexíveis que os compostos centrais, possibilitando o estudo de $\mathbf{k}$ fatores em vários níveis (cinco, sete ou nove). Aqueles considerados por nós nos quadros apresentam, todos, nove níveis.

Os delineamentos constantes do quadro 1 são ortogonais, divisíveis em blocos, e apresentam o nível do segundo fatorial quase sempre inferior a dois, condição que os torna bastante desejáveis na prática.

Os delineamentos constantes do quadro 2 são ortogonais, divisíveis em blocos e calculados impondo a condição de que $\alpha=\sqrt{\mathrm{k}}$ e $\gamma \alpha \approx \mathrm{W} \sqrt{\mathrm{k}}$, e contêm poucos pontos centrais (dois ou quatro). Em certo sentido, representam uma conjugação de dois delineamentos compostos centrais, com pontos pertencentes ao centro e a duas hiperesferas de raio $\sqrt{ } \mathbf{k}$ e $W / k$, sendo, cada um dos compostos centrais, do tipo quase D-ótimo.

Os delineamentos constantes do quadro 3 são ortogonais, rotacionais ou quase completamente rotacionais (no sentido dado por BOX \& HUNTER, 1), sendo também divisíveis, ortogonalmente, em dois, três ou cinco blocos; possibilitam uma estimação razoável do erro experimental e um teste eficiente de adequação do modelo. 
O delineamento composto central duplo possibilita, em pesquisas com fertilizante, estimar mais eficientemente a resposta nos anos bons $\mathrm{e}$ nos ruins, pois permitem, nos casos de "resposta em platô", estimar melhor os coeficientes, evitar-lhes $o$ viés, e analisar a parte central do

\section{delineamento como um composto central.}

Caberá ao pesquisador e ao estatístico decidir-se pela escolha do critério que julgar mais adequado, dependendo a escolha das características julgadas mais relevantes para o seu caso particular.

\section{"DOUBLE CENTRAL COMPOSTTE" DESIGNS}

\section{SUMMARY}

The present paper deals with the type of designs known as "Double Central Composite". Basically they are a symmetric type composed of two factorials (or fractional factorials) at levels \pm 1 and $\pm W$, two stars at levels $\pm \alpha$ and $\pm \gamma \alpha$ for each factor and central points, presenting five, seven or nine levels for each factor.

Due to the higher flexibility of this type of design in comparison with the central composite, properties like orthogonality of the coefficients, orthogonal blocking and rotatability are easily put together in the same design.

As these designs can be arranged with a fractionary part of a $2^{k}$ factorial, they can explore more levels in the range considered with just a few more points than the correspondent central composite design.

In agriculture, opposite to technological experiments, and principally in fertilizer research, due to the soil and climatic variability, the experiments should be performed with all treatments allocated at the same time. The responses obtained may be higher and enlarged in the good years and smaller or with a "plateau response" in the bad years. The double central composite design may fit better this situation, because by its structure they cover better a broader range of dosages.

If an adequate calibration was originally adopted and the year was a good one, the larger range will fit better the response. If the year was bad, the response is smaller and may present some type of "plateau response" to the higher dosages; the analysis of the complete design may cause a "bias" in the coefficients of the model and in the determination of the economical dosages. In this case the design makes possible to contour the problem by just analysing the central part of the design, as a central composite, using the levels \pm 1 for the factorial and $\pm \alpha$ to the axial, getting an "unbiased" estimate of the coefficients; for the same range of dosages originally utilized, this could not be obtained through an original central composite or with a $3 \times 3 \times 3$ factorial.

Basically this paper presents three types of design.

In Table 1, designs are presented that, besides being orthogonal, are orthogonally blocked in 2,3 or 5 blocks. The levels were chosen in such a manner as to become the $\mathrm{W}$ levels of all designs, smaller than two (levels higher than two are seldom used in practice).

The designs of the second group are orthogonal, orthogonally blocked with the external points all of them on the surface of two hyperspheres of rays $\alpha=\sqrt{\mathrm{k}}$ and $\gamma \alpha \approx W \sqrt{k}$, containing two or four central points; these designs may be considered as composed of two central composites, each one having, according with Lucas, quasi optimum D-efficiency.

In the third group, presented in Table 3, the designs are orthogonal, orthogonally blocked and with full rotatability or quasi rotatability (the last one presented with an asterisk), in Box and Hunter sense, with enough central points to furnish good estimates of the experimental error, allowing a more precise test for the adequacy of the model. 
The double central composite design should be of value for the research workers or for the statisticians in those cases in which blocking is very important as in field agriculture experiments and particularly in fertilizer experiments when we are looking for the economical response to NPK. They may be utilized in other areas of research with similar problems, in cases in which we want to evaluate the response for several levels of each factor.

\section{REFERENCIAS BIBLIOGRAFICAS}

1. BOX, G. E. P. \& HUNTER, J. S. Multifactor experimental designs for exploring response surfaces. Annals of Mathematical Statistics, 28:195-241, 1957.

2. — \& WILSON, K. B. On the experimental attainment of optimum conditions. Journal of the Royal Statistical Society B, 13:1-45, 1951.

3. CONAGIN, A. \& JORGE, J. P. N. Delineamento duplo central composto com 29 pontos. Bragantia, Campinas, 38:215-235, 1979.

4. —_ ; VENTURINI, W. R. Delineamentos experimentais utilizáveis na experimentação de campo. In: REYNAERT, E. E. La investigación de fertilidad de suelos para la producción en la zona templada. Montevideo, IICA. Zona Sur, 1969. p.171-182.

5. DAVIES, O. L. Design and analysis of industrial experiments. New York, Hafner Publishing Company, 1954. 637p.

6. HARTLEY, H. P. Smallest composite designs for quadratic response surfaces. Biometrics, 15:611-624, 1959.

7. LUCAS, J. M. Optimum composite designs. Technometrics, 16:561-567, 1974.

8. Which response surface design is best. Technometrics, 18:411-417, 1976.

9. MYERS, R. H. Response surface methodology. Boston, Allyn and Bacon, 1971. $243 p$.

10. VOSS, R. \& PESEK, J. T. Yield of corm as affected by fertilizer rates and environmental factors. Agronomy Journal, 59:567-572, 1967. 